\title{
DÉCOMPOSITION DE HELMHOLTZ PAR ONDELETTES : CONVERGENCE D'UN ALGORITHME ITÉRATIF*
}

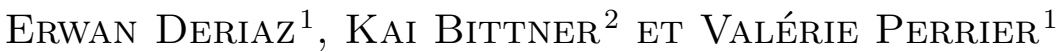

\begin{abstract}
Résumé. Dans ce qui suit, on présente des ondelettes à divergence nulle et des ondelettes à rotationnel nul, et on définit des projecteurs associés : ces projecteurs permettent de construire un algorithme itératif de décomposition de Helmholtz par ondelettes. Cette décomposition de Helmholtz est localisée en espace, contrairement à une décomposition de Helmholtz qui serait calculée par transformée de Fourier. On démontre la convergence de l'algorithme en dimensions 2 et 3, dans le cas particulier des ondelettes de Shannon.
\end{abstract}

\begin{abstract}
In what follows, we present tensor-product divergence-free and curl-free wavelets, and we define associated projectors. These projectors permit the construction of an iterative algorithm for the computation of the Helmholtz decomposition in terms of wavelets. This Helmholtz decomposition is localized in space, in contrast to a Helmholtz decomposition calculated by the Fourier transform. Finally, we show the convergence of the algorithm in 2 and 3 dimensions for the particular case of Shannon wavelets.
\end{abstract}

\section{INTRODUCTION}

Dans de nombreux problèmes, comme la simulation d'écoulements incompressibles (problème de Stokes, équations de Navier-Stokes [2,11]), ou en électromagnétisme (équations de Maxwell [10]), la solution doit vérifier une condition de divergence nulle. Lors de l'implémentation de méthodes numériques, il est souvent nécessaire de disposer d'un projecteur orthogonal sur l'ensemble des fonctions à divergence nulle.

La décomposition de Helmholtz $[2,6]$ consiste à décomposer un champ de vecteurs $\mathbf{u} \in\left(L^{2}\left(\mathbb{R}^{n}\right)\right)^{n}$ en somme de sa composante à divergence nulle $\mathbf{u}_{\text {div }}$ et de sa composante à rotationnel nul $\mathbf{u}_{\text {rot }}$. Plus précisément, il existe une fonction de courant $\psi$ et un potentiel $p$ tels que :

$$
\mathbf{u}=\mathbf{u}_{\mathrm{div}}+\mathbf{u}_{\mathrm{rot}}
$$

avec

$$
\mathbf{u}_{\mathrm{div}}=\operatorname{rot} \psi, \quad\left(\operatorname{div} \mathbf{u}_{\mathrm{div}}=0\right) \quad \text { et } \quad \mathbf{u}_{\mathrm{rot}}=\nabla p, \quad\left(\operatorname{rot} \mathbf{u}_{\mathrm{rot}}=0\right)
$$

De plus, les fonctions rot $\psi$ et $\nabla p$ sont orthogonales dans $\left(L^{2}\left(\mathbb{R}^{n}\right)\right)^{n}$, et les fonctions courant $\psi$ et potentiel $p$ sont uniques, à une constante additive près.

\footnotetext{
* Ce travail a reçu le soutien du projet européen HPRN-CT-2002-00286 "Breaking complexity".

${ }^{1}$ Laboratoire de Modélisation et Calcul de l'IMAG, BP 53, 38041 Grenoble cedex 9, France; e-mail : erwan.deriaz@imag.fr \& valerie.perrier@imag.fr

2 Université d'Ulm, Helmholtzstr. 18, D-89069 Ulm, Allemagne; e-mail : e-mail : kai.bittner@uni-ulm.de
}

(C) EDP Sciences, SMAI 2007 
Cette décomposition découle de la mise en somme directe orthogonale des espaces $\mathbf{H}_{\text {div } 0}\left(\mathbb{R}^{n}\right)$, l'espace des fonctions vectorielles à divergence nulle, et $\mathbf{H}_{\text {rot } 0}\left(\mathbb{R}^{n}\right)$ celui des fonctions vectorielles à rotationnel nul. En résumé :

$$
\left(L^{2}\left(\mathbb{R}^{n}\right)\right)^{n}=\mathbf{H}_{\operatorname{div} 0}\left(\mathbb{R}^{n}\right) \oplus^{\perp} \mathbf{H}_{\text {rot } 0}\left(\mathbb{R}^{n}\right)
$$

Cette décomposition est immédiate dans $\left(L^{2}\left(\mathbb{R}^{n}\right)\right)^{n}$, grâce au projecteur de Leray (projecteur orthogonal de $\left(L^{2}\left(\mathbb{R}^{n}\right)\right)^{n}$ dans $\left.\mathbf{H}_{\text {div } 0}\left(\mathbb{R}^{n}\right)\right)$ qui est explicite dans le domaine de Fourier. Sur des ouverts $\Omega$, la décomposition (1) est toujours vérifiée $[2,6]$.

On construit dans la partie 1 des bases d'ondelettes biorthogonales de $\mathbf{H}_{\text {div } 0}\left(\mathbb{R}^{n}\right)$ et $\mathbf{H}_{\text {rot } 0}\left(\mathbb{R}^{n}\right)$, et des projecteurs associés. Comme ces projecteurs sont obliques, on définit dans la partie 2 un algorithme itératif de décomposition de Helmholtz par ondelettes, dont on prouve la convergence en partie 3.

\section{Ondelettes À DiVergence nulle et À ROTATiOnNel Nul}

Les ondelettes à divergence nulle ont été définies par P.G. Lemarié en 1992 [8]. K. Urban les utilise pour la résolution du problème de Stokes [12], puis élargit la construction à des ondelettes à rotationnel nul [13]. Le principe fondamental de ces constructions repose sur l'existence de bases d'ondelettes biorthogonales (voir [3,9]) liées par différentiation. En particulier on utilisera le résultat suivant de [8] :

Proposition 1.1. Si $\left(V_{j}^{1}\right)$ est une analyse multirésolution $(A M R)$ de $L^{2}(\mathbb{R})$, d'ondelette associée $\psi_{1}$, alors il existe une $A M R\left(V_{j}^{0}\right)$, d'ondelette associée $\psi_{0}$, vérifiant :

$$
\psi_{1}^{\prime}(x)=\psi_{0}(x)
$$

On dispose ainsi de deux bases de Riesz de $\left.L^{2}(\mathbb{R}):\left(\psi_{1, j, k}(x)=2^{j / 2} \psi_{1}\left(2^{j} x-k\right)\right)\right)_{j, k \in \mathbb{Z}}$ et $\left(\psi_{0, j, k}\right)_{j, k \in \mathbb{Z}}$, reliées par dérivation.

Contrairement aux ondelettes proposées dans les travaux de Lemarié et de Urban, on préférera utiliser des bases d'ondelettes anisotropes à divergence nulle et à rotationnel nul, c'est-à-dire des produits tensoriels de bases d'ondelettes unidimensionnelles $[4,5]$. Nous reprenons ici les principaux ingrédients de leur définition, et des projecteurs associés. Ces constructions se généralisent en dimension $n$ quelconque d'espace [4].

\subsection{Ondelettes anisotropes à divergence nulle}

\subsubsection{Cas de la dimension 2}

En dimension 2, les ondelettes à divergence nulle anisotropes sont construites simplement en considérant le rotationnel des ondelettes de l'AMR tensorielle $\left(V_{j}^{1} \otimes V_{j}^{1}\right)$ :

$$
\Psi_{\operatorname{div} \mathbf{j}, \mathbf{k}}\left(x_{1}, x_{2}\right)=\operatorname{rot}\left(\psi_{1}\left(2^{j_{1}} x_{1}-k_{1}\right) \psi_{1}\left(2^{j_{2}} x_{2}-k_{2}\right)\right)=\mid \begin{aligned}
& 2^{j_{2}} \psi_{1}\left(2^{j_{1}} x_{1}-k_{1}\right) \psi_{0}\left(2^{j_{2}} x_{2}-k_{2}\right) \\
& -2^{j_{1}} \psi_{0}\left(2^{j_{1}} x_{1}-k_{1}\right) \psi_{1}\left(2^{j_{2}} x_{2}-k_{2}\right)
\end{aligned}
$$

$\mathbf{j}=\left(j_{1}, j_{2}\right) \in \mathbb{Z}^{2}$ est le paramètre d'échelle, et $\mathbf{k}=\left(k_{1}, k_{2}\right) \in \mathbb{Z}^{2}$ le paramètre de position. Quand $\mathbf{j}$ et $\mathbf{k}$ varient dans $\mathbb{Z}^{2}$, la famille $\left\{\Psi_{\text {div } \mathbf{j}, \mathbf{k}}\right\}$ forme une base de $\mathbf{H}_{\text {div } 0}\left(\mathbb{R}^{2}\right)$. Pour la compléter en une base de $\left(L^{2}\left(\mathbb{R}^{2}\right)\right)^{2}$, on introduit les fonctions :

$$
\Psi_{\mathrm{n} \mathbf{j}, \mathbf{k}}\left(x_{1}, x_{2}\right)=\mid \begin{aligned}
& 2^{j_{1}} \psi_{1}\left(2^{j_{1}} x_{1}-k_{1}\right) \psi_{0}\left(2^{j_{2}} x_{2}-k_{2}\right) \\
& 2^{j_{2}} \psi_{0}\left(2^{j_{1}} x_{1}-k_{1}\right) \psi_{1}\left(2^{j_{2}} x_{2}-k_{2}\right)
\end{aligned}
$$

en remarquant que pour $\mathbf{j}$ et $\mathbf{k}$ fixés, la fonction vectorielle $\Psi_{\mathrm{n} \mathbf{j}, \mathbf{k}}$ est orthogonale à $\Psi_{\operatorname{div} \mathbf{j}, \mathbf{k}}$. 
Si on veut décomposer un champ de vecteurs $\mathbf{u}$ dans cette nouvelle base, on part d'une décomposition classique de $\mathbf{u}$ en ondelettes tensorielles de l'AMR $\left(V_{j}^{1} \otimes V_{j}^{0}\right) \times\left(V_{j}^{0} \otimes V_{j}^{1}\right)$ :

$$
\mathbf{u}=\sum_{\mathbf{j} \in \mathbb{Z}^{2}} \sum_{\mathbf{k} \in \mathbb{Z}^{2}}\left(d_{1, \mathbf{j}, \mathbf{k}} \Psi_{\mathbf{j}, \mathbf{k}}^{1}+d_{2, \mathbf{j}, \mathbf{k}} \Psi_{\mathbf{j}, \mathbf{k}}^{2}\right)
$$

où on a noté, pour $\mathbf{j}, \mathbf{k} \in \mathbb{Z}^{2}$ :

$$
\begin{aligned}
& \Psi_{\mathbf{j}, \mathbf{k}}^{1}\left(x_{1}, x_{2}\right)=\mid \begin{array}{l}
\psi_{1}\left(2^{j_{1}} x_{1}-k_{1}\right) \psi_{0}\left(2^{j_{2}} x_{2}-k_{2}\right) \\
0
\end{array} \\
& \Psi_{\mathbf{j}, \mathbf{k}}^{2}\left(x_{1}, x_{2}\right)=\mid \begin{array}{l}
0 \\
\psi_{0}\left(2^{j_{1}} x_{1}-k_{1}\right) \psi_{1}\left(2^{j_{2}} x_{2}-k_{2}\right)
\end{array}
\end{aligned}
$$

les ondelettes anisotropes pour chaque composante (avec une normalisation $L^{\infty}$ ).

On écrit ensuite u sur la base d'ondelettes à divergence nulle et sur la base d'ondelettes complémentaires :

$$
\mathbf{u}=\sum_{\mathbf{j} \in \mathbb{Z}^{2}} \sum_{\mathbf{k} \in \mathbb{Z}^{2}}\left(d_{\operatorname{div} \mathbf{j}, \mathbf{k}} \Psi_{\operatorname{div} \mathbf{j}, \mathbf{k}}+d_{\mathrm{n} \mathbf{j}, \mathbf{k}} \Psi_{\mathrm{n} \mathbf{j}, \mathbf{k}}\right)
$$

ce qui conduit directement aux coefficients $d_{\operatorname{div} \mathbf{j}, \mathbf{k}}$ et $d_{\mathrm{n} \mathbf{j}, \mathbf{k}}$ :

$$
\left\{\begin{array}{c}
d_{\operatorname{div} \mathbf{j}, \mathbf{k}}=\frac{2^{j_{2}}}{2^{2 j_{1}}+2^{2 j_{2}}} d_{1, \mathbf{j}, \mathbf{k}}-\frac{2^{j_{1}}}{2^{2 j_{1}}+2^{2 j_{2}}} d_{2, \mathbf{j}, \mathbf{k}} \\
d_{\mathrm{n} \mathbf{j}, \mathbf{k}}=\frac{2^{j_{1}}}{2^{2 j_{1}}+2^{2 j_{2}}} d_{1, \mathbf{j}, \mathbf{k}}+\frac{2^{j_{2}}}{2^{2 j_{1}}+2^{2 j_{2}}} d_{2, \mathbf{j}, \mathbf{k}}
\end{array}\right.
$$

Remarque 1.2. Le choix des ondelettes complémentaires $\Psi_{n \mathbf{j}, \mathbf{k}}$ n'est pas unique, et ce choix conditionne le calcul des coefficients $d_{\text {div } \mathbf{j}, \mathbf{k}}$ et $d_{\mathrm{n} \mathbf{j}, \mathbf{k}}$. Nous verrons à la section 4 que ce choix conditionne également la convergence de l'algorithme de décomposition de Helmholtz par ondelettes. Bien sûr si u est à divergence nulle, on retrouve $d_{\mathbf{n} \mathbf{j}, \mathbf{k}}=0$.

\subsubsection{Cas de la dimension 3}

Comme en dimension 2, les ondelettes à divergence nulle anisotropes sont construites en considérant le rotationnel d'ondelettes anisotropes standard :

$$
\begin{aligned}
& \Psi_{\operatorname{div} 1, \mathbf{j}, \mathbf{k}}\left(x_{1}, x_{2}, x_{3}\right)=\operatorname{rot}\left|\begin{array}{l}
\psi_{0} \psi_{1} \psi_{1} \\
0 \\
0
\end{array}=\right| \begin{array}{l}
0 \\
2^{j_{3}} \psi_{0}\left(2^{j_{1}} x_{1}-k_{1}\right) \psi_{1}\left(2^{j_{2}} x_{2}-k_{2}\right) \psi_{0}\left(2^{j_{3}} x_{3}-k_{3}\right) \\
-2^{j_{2}} \psi_{0}\left(2^{j_{1}} x_{1}-k_{1}\right) \psi_{0}\left(2^{j_{2}} x_{2}-k_{2}\right) \psi_{1}\left(2^{j_{3}} x_{3}-k_{3}\right) \\
-k
\end{array} \\
& \Psi_{\operatorname{div} 2, \mathbf{j}, \mathbf{k}}\left(x_{1}, x_{2}, x_{3}\right)=\operatorname{rot}\left|\begin{array}{l}
0 \\
\psi_{1} \psi_{0} \psi_{1} \\
0
\end{array}=\right| \begin{array}{l}
-2^{j_{3}} \psi_{1}\left(2^{j_{1}} x_{1}-k_{1}\right) \psi_{0}\left(2^{j_{2}} x_{2}-k_{2}\right) \psi_{0}\left(2^{j_{3}} x_{3}-k_{3}\right) \\
0 \\
2^{j_{1}} \psi_{0}\left(2^{j_{1}} x_{1}-k_{1}\right) \psi_{0}\left(2^{j_{2}} x_{2}-k_{2}\right) \psi_{1}\left(2^{j_{3}} x_{3}-k_{3}\right)
\end{array} \\
& \Psi_{\operatorname{div} 3, \mathbf{j}, \mathbf{k}}\left(x_{1}, x_{2}, x_{3}\right)=\operatorname{rot}\left|\begin{array}{l}
0 \\
0 \\
\psi_{1} \psi_{1} \psi_{0}
\end{array}=\right| \begin{array}{l}
2^{j_{2}} \psi_{1}\left(2^{j_{1}} x_{1}-k_{1}\right) \psi_{0}\left(2^{j_{2}} x_{2}-k_{2}\right) \psi_{0}\left(2^{j_{3}} x_{3}-k_{3}\right) \\
-2^{j_{1}} \psi_{0}\left(2^{j_{1}} x_{1}-k_{1}\right) \psi_{1}\left(2^{j_{2}} x_{2}-k_{2}\right) \psi_{0}\left(2^{j_{3}} x_{3}-k_{3}\right) \\
0
\end{array} \mid
\end{aligned}
$$

Comme c'est une famille liée $\left(2^{j_{1}} \Psi_{\operatorname{div} 1, \mathbf{j}, \mathbf{k}}+2^{j_{2}} \Psi_{\operatorname{div} 2, \mathbf{j}, \mathbf{k}}+2^{j_{3}} \Psi_{\operatorname{div} 3, \mathbf{j}, \mathbf{k}}=0\right)$, il faut choisir deux fonctions parmi les trois ci-dessus pour engendrer une base de $\mathbf{H}_{\text {div } 0}\left(\mathbb{R}^{3}\right)$. Dans tous les cas, on choisit comme fonction 
complémentaire celle orthogonale aux trois autres :

$$
\Psi_{\mathrm{n} \mathbf{j}, \mathbf{k}}\left(x_{1}, x_{2}, x_{3}\right)=\mid \begin{aligned}
& 2^{j_{1}} \psi_{1}\left(2^{j_{1}} x_{1}-k_{1}\right) \psi_{0}\left(2^{j_{2}} x_{2}-k_{2}\right) \psi_{0}\left(2^{j_{3}} x_{3}-k_{3}\right) \\
& 2^{j_{2}} \psi_{0}\left(2^{j_{1}} x_{1}-k_{1}\right) \psi_{1}\left(2^{j_{2}} x_{2}-k_{2}\right) \psi_{0}\left(2^{j_{3}} x_{3}-k_{3}\right) \\
& 2^{j_{3}} \psi_{0}\left(2^{j_{1}} x_{1}-k_{1}\right) \psi_{0}\left(2^{j_{2}} x_{2}-k_{2}\right) \psi_{1}\left(2^{j_{3}} x_{3}-k_{3}\right)
\end{aligned}
$$

Pour calculer la décomposition d'un champ de vecteurs sur cette nouvelle base, on introduit de nouveau les ondelettes tensorielles, composante par composante, de l'AMR $\left(V_{j}^{1} \otimes V_{j}^{0} \otimes V_{j}^{0}\right) \times\left(V_{j}^{0} \otimes V_{j}^{1} \otimes V_{j}^{0}\right) \times\left(V_{j}^{0} \otimes V_{j}^{0} \otimes V_{j}^{1}\right)$ :

$$
\begin{aligned}
& \Psi_{\mathbf{j}, \mathbf{k}}^{1}\left(x_{1}, x_{2}, x_{3}\right)=\mid \begin{array}{l}
\psi_{1}\left(2^{j_{1}} x_{1}-k_{1}\right) \psi_{0}\left(2^{j_{2}} x_{2}-k_{2}\right) \psi_{0}\left(2^{j_{3}} x_{3}-k_{3}\right) \\
0
\end{array} \\
& \Psi_{\mathbf{j}, \mathbf{k}}^{2}\left(x_{1}, x_{2}, x_{3}\right)= \\
& \begin{array}{l}
0 \\
\psi_{0}\left(2^{j_{1}} x_{1}-k_{1}\right) \psi_{1}\left(2^{j_{2}} x_{2}-k_{2}\right) \psi_{0}\left(2^{j_{3}} x_{3}-k_{3}\right) \\
0
\end{array} \\
& \Psi_{\mathbf{j}, \mathbf{k}}^{3}\left(x_{1}, x_{2}, x_{3}\right)=\mid \begin{array}{l}
0 \\
0 \\
\psi_{0}\left(2^{j_{1}} x_{1}-k_{1}\right) \psi_{0}\left(2^{j_{2}} x_{2}-k_{2}\right) \psi_{1}\left(2^{j_{3}} x_{3}-k_{3}\right)
\end{array}
\end{aligned}
$$

avec $\mathbf{j}=\left(j_{1}, j_{2}, j_{3}\right) \in \mathbb{Z}^{3}$ et $\mathbf{k}=\left(k_{1}, k_{2}, k_{3}\right) \in \mathbb{Z}^{3}$.

On passe alors de la décomposition de $\mathbf{u}$, sur ces ondelettes classiques :

$$
\mathbf{u}=\sum_{i=1}^{3} \sum_{\mathbf{j} \in \mathbb{Z}^{3}} \sum_{\mathbf{k} \in \mathbb{Z}^{3}} d_{i \mathbf{j}, \mathbf{k}} \Psi_{\mathbf{j}, \mathbf{k}}^{i}
$$

à la décomposition sur les ondelettes vecteurs à divergence nulle et leurs complémentaires :

$$
\mathbf{u}=\sum_{\mathbf{j} \in \mathbb{Z}^{3}} \sum_{\mathbf{k} \in \mathbb{Z}^{3}}\left(d_{\operatorname{div} 1, \mathbf{j}, \mathbf{k}} \Psi_{\operatorname{div} 1, \mathbf{j}, \mathbf{k}}+d_{\operatorname{div} 2, \mathbf{j}, \mathbf{k}} \Psi_{\operatorname{div} 2, \mathbf{j}, \mathbf{k}}+d_{\operatorname{div} 3, \mathbf{j}, \mathbf{k}} \Psi_{\operatorname{div} 3, \mathbf{j}, \mathbf{k}}+d_{\mathrm{n} \mathbf{j}, \mathbf{k}} \Psi_{\mathrm{n} \mathbf{j}, \mathbf{k}}\right)
$$

en ajoutant comme condition

$$
2^{j_{1}} d_{\operatorname{div} 1, \mathbf{j}, \mathbf{k}}+2^{j_{2}} d_{\operatorname{div} 2, \mathbf{j}, \mathbf{k}}+2^{j_{3}} d_{\operatorname{div} 3, \mathbf{j}, \mathbf{k}}=0
$$

pour avoir autant d'équations que d'inconnues, et pour que la matrice de changement de coordonnées soit orthogonale [4]. On obtient alors :

$$
\begin{cases}d_{\operatorname{div} 1, \mathbf{j}, \mathbf{k}}=\frac{2^{j_{3}} d_{2, \mathbf{j}, \mathbf{k}}-2^{j_{2}} d_{3, \mathbf{j}, \mathbf{k}}}{2^{2 j}+2^{2 j_{2}}+2^{2 j_{3}}} & d_{\text {div } 2, \mathbf{j}, \mathbf{k}}=\frac{-2^{j_{3}} d_{1, \mathbf{j}, \mathbf{k}}+2^{j_{1}} d_{3, \mathbf{j}, \mathbf{k}}}{2^{2 j_{1}}+2^{2 j_{2}}+2^{2 j_{3}}} \\ d_{\operatorname{div} 3, \mathbf{j}, \mathbf{k}}=\frac{2^{j_{2}} d_{1, \mathbf{j}, \mathbf{k}}-2^{j_{1}} d_{2, \mathbf{j}, \mathbf{k}}}{2^{2 j_{1}}+2^{2 j_{2}}+2^{2 j_{3}}} & d_{\mathrm{n} \mathbf{j}, \mathbf{k}}=\frac{2^{j_{1}} d_{1, \mathbf{j}, \mathbf{k}}+2^{j_{2}} d_{2, \mathbf{j}, \mathbf{k}}+2^{j_{3}} d_{3, \mathbf{j}, \mathbf{k}}}{2^{2 j_{1}}+2^{2 j_{2}}+2^{2 j_{3}}}\end{cases}
$$

\subsection{Ondelettes anisotropes à rotationnel nul}

En dimension 2, on construit des ondelettes à rotationnel nul en considérant le gradient des ondelettes tensorielles de l'AMR $\left(V_{j}^{1} \otimes V_{j}^{1}\right)$ :

$$
\Psi_{\operatorname{rot} \mathbf{j}, \mathbf{k}}\left(x_{1}, x_{2}\right)=\nabla\left(\psi_{1}\left(2^{j_{1}} x_{1}-k_{1}\right) \psi_{1}\left(2^{j_{2}} x_{2}-k_{2}\right)\right)=\mid \begin{aligned}
& 2^{j_{1}} \psi_{0}\left(2^{j_{1}} x_{1}-k_{1}\right) \psi_{1}\left(2^{j_{2}} x_{2}-k_{2}\right) \\
& 2^{j_{2}} \psi_{1}\left(2^{j_{1}} x_{1}-k_{1}\right) \psi_{0}\left(2^{j_{2}} x_{2}-k_{2}\right)
\end{aligned}
$$


qui engendrent une base de l'espace $\mathbf{H}_{\text {rot } 0}\left(\mathbb{R}^{2}\right)$, et que l'on complète en une base de $\left(L^{2}\left(\mathbb{R}^{2}\right)\right)^{2}$ avec les fonctions :

$$
\Psi_{\mathrm{N} \mathbf{j}, \mathbf{k}}\left(x_{1}, x_{2}\right)=\mid \begin{aligned}
& 2^{j_{2}} \psi_{0}\left(2^{j_{1}} x_{1}-k_{1}\right) \psi_{1}\left(2^{j_{2}} x_{2}-k_{2}\right) \\
& -2^{j_{1}} \psi_{1}\left(2^{j_{1}} x_{1}-k_{1}\right) \psi_{0}\left(2^{j_{2}} x_{2}-k_{2}\right)
\end{aligned}
$$

La décomposition sur ces ondelettes s'obtient en partant de la décomposition standard :

$$
\mathbf{u}=\sum_{\mathbf{j} \in \mathbb{Z}^{2}} \sum_{\mathbf{k} \in \mathbb{Z}^{2}}\left(d_{1, \mathbf{j}, \mathbf{k}} \Psi_{1, \mathbf{j}, \mathbf{k}}^{\#}+d_{2, \mathbf{j}, \mathbf{k}} \Psi_{2, \mathbf{j}, \mathbf{k}}^{\#}\right)
$$

sur les ondelettes canoniques, composante par composante :

$$
\Psi_{1, \mathbf{j}, \mathbf{k}}^{\#}\left(x_{1}, x_{2}\right)=\left|\begin{array}{l}
\psi_{0}\left(2^{j_{1}} x_{1}-k_{1}\right) \psi_{1}\left(2^{j_{2}} x_{2}-k_{2}\right) \\
0
\end{array} \Psi_{2, \mathbf{j}, \mathbf{k}}^{\#}\left(x_{1}, x_{2}\right)=\right| \begin{aligned}
& 0 \\
& \psi_{1}\left(2^{j_{1}} x_{1}-k_{1}\right) \psi_{0}\left(2^{j_{2}} x_{2}-k_{2}\right)
\end{aligned}
$$

On écrit alors la nouvelle décomposition :

$$
\mathbf{u}=\sum_{\mathbf{j} \in \mathbb{Z}^{2}} \sum_{\mathbf{k} \in \mathbb{Z}^{2}}\left(d_{\operatorname{rot} \mathbf{j}, \mathbf{k}} \Psi_{\operatorname{rot} \mathbf{j}, \mathbf{k}}+d_{\mathrm{N} \mathbf{j}, \mathbf{k}} \Psi_{\mathrm{N} \mathbf{j}, \mathbf{k}}\right)
$$

avec les coefficients :

$$
\left\{\begin{array}{l}
d_{\text {rot } \mathbf{j}, \mathbf{k}}=\frac{2^{j_{1}}}{2^{2 j_{1}}+2^{2 j_{2}}} d_{1, \mathbf{j}, \mathbf{k}}+\frac{2^{j_{2}}}{2^{2 j_{1}}+2^{2 j_{2}}} d_{2, \mathbf{j}, \mathbf{k}} \\
d_{\mathrm{N} \mathbf{j}, \mathbf{k}}=\frac{2^{j_{2}}}{2^{2 j_{1}}+2^{2 j_{2}}} d_{1, \mathbf{j}, \mathbf{k}}-\frac{2^{j_{1}}}{2^{2 j_{1}}+2^{2 j_{2}}} d_{2, \mathbf{j}, \mathbf{k}}
\end{array}\right.
$$

De la même manière, en dimension 3, les ondelettes à rotationnel nul sont construites en considérant le gradient des ondelettes tensorielles de l'AMR $\left(V_{j}^{1} \otimes V_{j}^{1} \otimes V_{j}^{1}\right)$ :

$$
\begin{aligned}
\Psi_{\text {rot } \mathbf{j}, \mathbf{k}}\left(x_{1}, x_{2}\right)= & \nabla\left(\psi_{1}\left(2^{j_{1}} x_{1}-k_{1}\right) \psi_{1}\left(2^{j_{2}} x_{2}-k_{2}\right) \psi_{1}\left(2^{j_{3}} x_{3}-k_{3}\right)\right) \\
= & \mid \begin{array}{l}
2^{j_{1}} \psi_{0}\left(2^{j_{1}} x_{1}-k_{1}\right) \psi_{1}\left(2^{j_{2}} x_{2}-k_{2}\right) \psi_{1}\left(2^{j_{3}} x_{3}-k_{3}\right) \\
2^{j_{2}} \psi_{1}\left(2^{j_{1}} x_{1}-k_{1}\right) \psi_{0}\left(2^{j_{2}} x_{2}-k_{2}\right) \psi_{1}\left(2^{j_{3}} x_{3}-k_{3}\right) \\
2^{j_{3}} \psi_{1}\left(2^{j_{1}} x_{1}-k_{1}\right) \psi_{1}\left(2^{j_{2}} x_{2}-k_{2}\right) \psi_{0}\left(2^{j_{3}} x_{3}-k_{3}\right)
\end{array}
\end{aligned}
$$

qui engendrent une base de l'espace $\mathbf{H}_{\text {rot } 0}\left(\mathbb{R}^{3}\right)$, et que l'on complète en une famille génératrice de $\left(L^{2}\left(\mathbb{R}^{3}\right)\right)^{3}$ avec les fonctions (liées car $\sum_{i=1}^{3} 2^{j_{i}} \Psi_{\mathrm{N} i, \mathbf{j}, \mathbf{k}}=0$ ) :

$$
\begin{aligned}
\Psi_{\mathrm{N} 1, \mathbf{j}, \mathbf{k}}\left(x_{1}, x_{2}, x_{3}\right)= & \mid \begin{array}{l}
0 \\
-2^{j_{3}} \psi_{1}\left(2^{j_{1}} x_{1}-k_{1}\right) \psi_{0}\left(2^{j_{2}} x_{2}-k_{2}\right) \psi_{1}\left(2^{j_{3}} x_{3}-k_{3}\right) \\
2^{j_{2}} \psi_{1}\left(2^{j_{1}} x_{1}-k_{1}\right) \psi_{1}\left(2^{j_{2}} x_{2}-k_{2}\right) \psi_{0}\left(2^{j_{3}} x_{3}-k_{3}\right)
\end{array} \\
\Psi_{\mathrm{N} 2, \mathbf{j}, \mathbf{k}}\left(x_{1}, x_{2}, x_{3}\right)= & \begin{array}{l}
2^{j_{3}} \psi_{0}\left(2^{j_{1}} x_{1}-k_{1}\right) \psi_{1}\left(2^{j_{2}} x_{2}-k_{2}\right) \psi_{1}\left(2^{j_{3}} x_{3}-k_{3}\right) \\
0 \\
-2^{j_{1}} \psi_{1}\left(2^{j_{1}} x_{1}-k_{1}\right) \psi_{1}\left(2^{j_{2}} x_{2}-k_{2}\right) \psi_{0}\left(2^{j_{3}} x_{3}-k_{3}\right) \\
-2^{j_{2}} \psi_{0}\left(2^{j_{1}} x_{1}-k_{1}\right) \psi_{1}\left(2^{j_{2}} x_{2}-k_{2}\right) \psi_{1}\left(2^{j_{3}} x_{3}-k_{3}\right) \\
2^{j_{1}} \psi_{1}\left(2^{j_{1}} x_{1}-k_{1}\right) \psi_{0}\left(2^{j_{2}} x_{2}-k_{2}\right) \psi_{1}\left(2^{j_{3}} x_{3}-k_{3}\right) \\
0
\end{array}
\end{aligned}
$$

\section{Décomposition de Helmholtz par ondelettes : PRÉSEntation D'Un ALGORITHME ITÉRATIF}

L'objectif est maintenant de décomposer un champ de vecteurs $\mathbf{u}$, en ondelettes à divergence nulle et en ondelettes à rotationnel nul. Plus précisement, on souhaite réécrire l'équation (1) ( $\mathbb{P}$ désignant le projecteur de 
Leray, et $\mathbb{Q}$ le projecteur orthogonal sur les fonctions vectorielles à rotationnel nul) :

$$
\mathbf{u}=\mathbb{P} \mathbf{u}+\mathbb{Q} \mathbf{u}, \quad \mathbb{P} \mathbf{u}=\mathbf{u}_{\mathrm{div}}, \quad \mathbb{Q} \mathbf{u}=\mathbf{u}_{\text {rot }}
$$

où $\mathbf{u}_{\text {div }}$ et $\mathbf{u}_{\text {rot }}$ sont cherchés sous la forme de leurs décompositions en ondelettes à divergence nulle et à rotationnel nul :

$$
\mathbf{u}_{\mathrm{div}}=\mathbb{P} \mathbf{u}=\sum_{\mathbf{j}, \mathbf{k}} d_{\operatorname{div} \mathbf{j}, \mathbf{k}} \Psi_{\operatorname{div} \mathbf{j}, \mathbf{k}} \quad \text { et } \quad \mathbf{u}_{\mathrm{rot}}=\mathbb{Q} \mathbf{u}=\sum_{\mathbf{j}, \mathbf{k}} d_{\operatorname{rot} \mathbf{j}, \mathbf{k}} \Psi_{\mathrm{rot}} \mathbf{j}, \mathbf{k}
$$

Cependant les bases d'ondelettes à divergence nulle, comme les bases d'ondelettes à rotationnel nul, ne sont pas des bases orthogonales, et les projecteurs associés sont des projecteurs obliques qui dépendent du choix des espaces complémentaires $H_{\mathrm{n}}=\operatorname{Span}\left\{\Psi_{\mathrm{n}}\right\}$ et $\mathrm{H}_{\mathrm{N}}=\operatorname{Span}\left\{\Psi_{\mathrm{N}}\right\}$ introduits à la section 2 .

On dispose des décompositions d'espaces non orthogonales suivantes :

$$
\left(L^{2}\left(\mathbb{R}^{n}\right)\right)^{n}=\mathbf{H}_{\text {div } 0} \oplus \mathrm{H}_{\mathrm{n}} \quad \text { et } \quad\left(L^{2}\left(\mathbb{R}^{n}\right)\right)^{n}=\mathrm{H}_{\mathrm{N}} \oplus \mathbf{H}_{\text {rot } 0}
$$

et on note, pour un champ de vecteurs $\mathbf{u} \in\left(L^{2}\left(\mathbb{R}^{n}\right)\right)^{n}$ :

$$
\mathbf{u}=\mathrm{P}_{\mathrm{div}} \mathbf{u}+\mathrm{Q}_{\mathrm{n}} \mathbf{u}
$$

la décomposition en ondelettes à divergence nulle plus le supplémentaire dans $\mathrm{H}_{\mathrm{n}}$, puis

$$
\mathbf{u}=\mathrm{P}_{\mathrm{N}} \mathbf{u}+\mathrm{Q}_{\mathrm{rot}} \mathbf{u}
$$

la décomposition en ondelettes à rotationnel nul plus le supplémentaire dans $\mathrm{H}_{\mathrm{N}}$. La dimension d'espace $n$ est quelconque, mais on ne va traiter ici que les cas $n=2$ et 3 .

\section{Construction itérative des composantes à divergence nulle et à rotationnel nul du champ}

Disposant des deux décompositions (13) et (14), une permettant d'extraire imparfaitement la partie à divergence nulle du champ et l'autre la partie à rotationnel nul, on se propose de les alterner de façon itérative, jusqu'à convergence du résidu vers 0 . On s'attend alors à un processus de convergence comme schématisé à la figure 1.

En tenant compte des notations définies précédemment et en partant de $\mathbf{u}^{0}=\mathbf{u}$, la première étape de l'algorithme s'écrit :

$$
\left\{\begin{array}{l}
\mathbf{u}^{1 / 2}=\mathbf{u}^{0}-\mathrm{P}_{\mathrm{div}} \mathbf{u}^{0}=\mathrm{Q}_{\mathrm{n}} \mathbf{u}^{0} \\
\mathbf{u}^{1}=\mathbf{u}^{1 / 2}-\mathrm{Q}_{\mathrm{rot}} \mathbf{u}^{1 / 2}=\mathrm{P}_{\mathrm{N}} \mathbf{u}^{1 / 2}
\end{array}\right.
$$

puis se généralise à :

$$
\left\{\begin{array}{l}
\mathbf{u}^{1}=\mathrm{P}_{\mathrm{N}} \mathrm{Q}_{\mathrm{n}} \mathbf{u}^{0} \\
\mathbf{u}^{p+1}=\mathrm{P}_{\mathrm{N}} \mathrm{Q}_{\mathrm{n}} \mathbf{u}^{p} \quad \forall p \geq 1
\end{array}\right.
$$

La suite $\mathbf{u}^{p}$ ainsi définie vérifie :

$$
\mathbf{u}^{p}=\underbrace{\mathrm{P}_{\mathrm{div}} \mathbf{u}^{p}}_{\mathbf{u}_{\mathrm{div}}^{p}}+\underbrace{\mathrm{Q}_{\mathrm{rot}} \mathrm{Q}_{\mathrm{n}} \mathbf{u}^{p}}_{\mathbf{u}_{\mathrm{rot}}^{p}}+\underbrace{\mathrm{P}_{\mathrm{N}} \mathrm{Q}_{\mathrm{n}} \mathbf{u}^{p}}_{\mathbf{u}^{p+1}}
$$




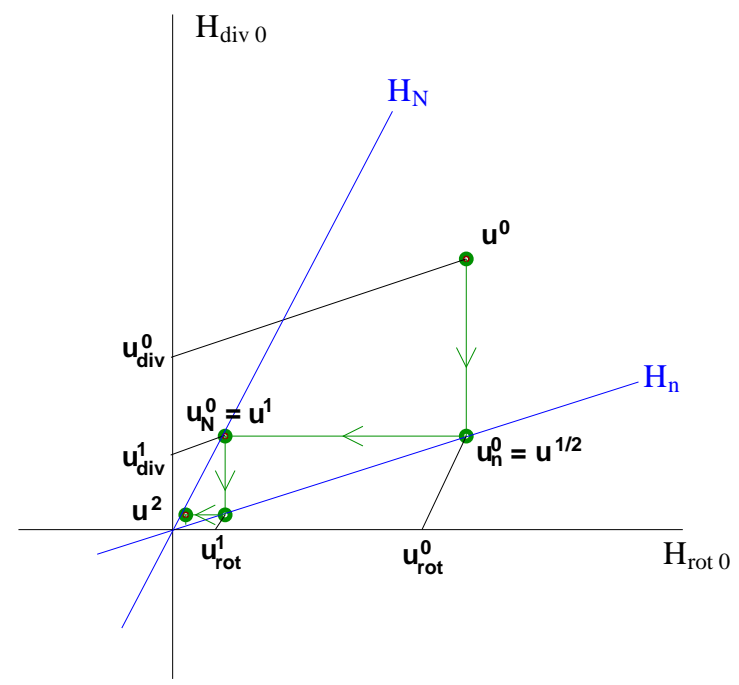

FIGURE 1. Schéma idéalisé du processus de convergence de l'algorithme de Helmholtz par ondelettes avec $H_{n}=\operatorname{vect}\left\{\Psi_{n \mathbf{j}, \mathbf{k}}\right\}$ et $H_{N}=\operatorname{vect}\left\{\Psi_{N \mathbf{j}, \mathbf{k}}\right\}$.

À la limite, si la suite $\left(\mathbf{u}^{p}\right)_{p \in \mathbb{N}}$ converge vers 0 , on obtient la décomposition (10) avec :

$$
\mathbf{u}_{\mathrm{div}}=\sum_{p=0}^{+\infty} \mathbf{u}_{\mathrm{div}}^{p} \quad \text { et } \quad \mathbf{u}_{\mathrm{rot}}=\sum_{p=0}^{+\infty} \mathbf{u}_{\mathrm{rot}}^{p}
$$

Idéalement, cet algorithme converge de la même façon que la suite $\left(\mathbf{u}^{p}\right)$ tend vers 0 dans le schéma de la figure 1 : dans le chapitre suivant, la convergence est démontrée dans les cas bi et tridimensionnels, et pour le cas particulier des ondelettes de Shannon qui sont à support physique infini mais à localisation optimale en Fourier. Cette convergence a aussi été testée et observée sur des champs divers et variés (réguliers, irréguliers, aléatoires ou issus de simulations) 2D et 3D avec des ondelettes splines [4].

Cependant, une rapide analyse de la figure 1 donne une bonne idée de ce qui va être crucial dans cette convergence : la proximité de l'espace $H_{\mathrm{n}}$ engendré par la famille $\left\{\Psi_{\mathbf{n} \mathbf{j}, \mathbf{k}}\right\}$, et celle de l'espace $H_{\mathrm{N}}$ engendré par $\left\{\Psi_{\mathrm{N} \mathbf{j}, \mathbf{k}}\right\}$ avec respectivement les espaces $\mathrm{H}_{\text {rot } 0}$ et $\mathrm{H}_{\text {div } 0}$.

\section{Preuves de la Convergence}

On procéde en deux temps. Tout d'abord on énonce un théorème général de convergence : on peut voir qu'un critère simple portant sur les espaces $\mathrm{H}_{n}$ et $\mathrm{H}_{\mathrm{N}}$ permet d'aboutir à la convergence de l'algorithme. Le principe est de dire que si $\mathrm{H}_{n}$ est toujours plus proche de $\mathrm{H}_{\text {rot } 0}$ que de $\mathrm{H}_{\text {div } 0}$, et si $\mathrm{H}_{\mathrm{N}}$ est toujours plus proche de $\mathrm{H}_{\text {div } 0}$ que de $\mathrm{H}_{\text {rot } 0}$, alors l'algorithme converge. Dans un deuxième temps, on montre que les hypothèses de ce théorème sont vérifiées dans le cas des ondelettes de Shannon.

\subsection{Convergence de l'algorithme}

Théorème 3.1. S'il existe deux réels strictement positifs $q_{\mathrm{n}}, q_{\mathrm{N}}$ tels que :

$$
\forall \mathbf{f}_{\mathrm{n}} \in \mathrm{H}_{\mathrm{n}}, \quad\left\|\mathbb{P} \mathbf{f}_{\mathrm{n}}\right\|_{L^{2}} \leq q_{\mathrm{n}}\left\|\mathbb{Q} \mathbf{f}_{\mathrm{n}}\right\|_{L^{2}}
$$

et

$$
\forall \mathbf{f}_{\mathrm{N}} \in \mathrm{H}_{\mathrm{N}}, \quad\left\|\mathbb{Q} \mathbf{f}_{\mathrm{N}}\right\|_{L^{2}} \leq q_{\mathrm{N}}\left\|\mathbb{P} \mathbf{f}_{\mathrm{N}}\right\|_{L^{2}}
$$


alors

$$
\forall \mathbf{f}_{\mathrm{N}} \in \mathrm{H}_{\mathrm{N}}, \quad\left\|\mathrm{Q}_{\mathrm{n}} \mathbf{f}_{\mathrm{N}}\right\|_{L^{2}} \leq \sqrt{\frac{1+q_{\mathrm{n}}^{2}}{1+q_{\mathrm{N}}^{2}}} q_{\mathrm{N}}\left\|\mathbf{f}_{\mathrm{N}}\right\|_{L^{2}}
$$

et

$$
\forall \mathbf{f}_{\mathrm{n}} \in \mathrm{H}_{\mathrm{n}}, \quad\left\|\mathrm{P}_{\mathrm{N}} \mathbf{f}_{\mathrm{n}}\right\|_{L^{2}} \leq \sqrt{\frac{1+q_{\mathrm{N}}^{2}}{1+q_{\mathrm{n}}^{2}}} q_{\mathrm{n}}\left\|\mathbf{f}_{\mathrm{n}}\right\|_{L^{2}}
$$

On en déduit alors que la suite $\left(\mathbf{u}^{p}\right)_{p \in \mathbb{N}}$ définie par la formule (15) vérifie :

$$
\left\|\mathbf{u}^{p+1}\right\|_{L^{2}} \leq q_{\mathrm{n}} q_{\mathrm{N}}\left\|\mathbf{u}^{p}\right\|_{L^{2}}
$$

Si bien que, si le produit $q_{\mathrm{n}} \times q_{\mathrm{N}}$ est strictement inférieur à 1 , la suite décroît exponentiellement.

Démonstration. Soit $\mathbf{f}_{\mathrm{N}} \in \mathrm{H}_{\mathrm{N}}$.

Comme $\mathrm{P}_{\text {div }} \mathbf{f}_{\mathrm{N}} \in \mathrm{H}_{\text {div } 0}$, on a $\mathbb{Q} \mathrm{P}_{\text {div }} \mathbf{f}_{\mathrm{N}}=0$ et $\mathbb{Q} \mathrm{Q}_{\mathrm{n}} \mathbf{f}_{\mathrm{N}}=\mathbb{Q}\left(\mathbf{f}_{\mathrm{N}}-\mathrm{P}_{\text {div }} \mathbf{f}_{\mathrm{N}}\right)=\mathbb{Q} \mathbf{f}_{\mathrm{N}}$.

Et comme $Q_{\mathrm{n}} \mathbf{f}_{\mathrm{N}} \in \mathrm{H}_{\mathrm{n}}$, d'après l'hypothèse (16),

$$
\left\|\mathbb{P} Q_{\mathrm{n}} \mathbf{f}_{\mathrm{N}}\right\|_{L^{2}} \leq q_{\mathrm{n}}\left\|\mathbb{Q} \mathrm{Q}_{\mathrm{n}} \mathbf{f}_{\mathrm{N}}\right\|_{L^{2}}=q_{\mathrm{n}}\left\|\mathbb{Q} \mathbf{f}_{\mathrm{N}}\right\|_{L^{2}}
$$

Si bien que dans $L^{2}$,

$$
\left\|\mathrm{Q}_{\mathrm{n}} \mathbf{f}_{\mathrm{N}}\right\|^{2}=\left\|\mathbb{Q} \mathrm{Q}_{\mathrm{n}} \mathbf{f}_{\mathrm{N}}\right\|^{2}+\left\|\mathbb{P} \mathrm{Q}_{\mathrm{n}} \mathbf{f}_{\mathrm{N}}\right\|^{2} \leq\left(1+q_{\mathrm{n}}^{2}\right)\left\|\mathbb{Q} \mathbf{f}_{\mathrm{N}}\right\|^{2}
$$

Par ailleurs, d'après l'hypothèse (17), on a $\left\|\mathbb{Q} \mathbf{f}_{\mathrm{N}}\right\| \leq q_{\mathrm{N}}\left\|\mathbb{P} \mathbf{f}_{\mathrm{N}}\right\|$ et donc

$$
\left\|\mathbf{f}_{\mathrm{N}}\right\|^{2}=\left\|\mathbb{P} \mathbf{f}_{\mathrm{N}}\right\|^{2}+\left\|\mathbb{Q} \mathbf{f}_{\mathrm{N}}\right\|^{2} \geq \frac{1}{q_{\mathrm{N}}^{2}}\left\|\mathbb{Q} \mathbf{f}_{\mathrm{N}}\right\|^{2}+\left\|\mathbb{Q} \mathbf{f}_{\mathrm{N}}\right\|^{2}=\frac{1+q_{\mathrm{N}}^{2}}{q_{\mathrm{N}}^{2}}\left\|\mathbb{Q} \mathbf{f}_{\mathrm{N}}\right\|^{2} \geq \frac{1+q_{\mathrm{N}}^{2}}{q_{\mathrm{N}}^{2}} \frac{1}{1+q_{\mathrm{n}}^{2}}\left\|\mathrm{Q}_{\mathrm{n}} \mathbf{f}_{\mathrm{N}}\right\|^{2}
$$

D'où finalement

$$
\left\|\mathrm{Q}_{\mathrm{n}} \mathbf{f}_{\mathrm{N}}\right\|^{2} \leq \frac{q_{\mathrm{N}}^{2}\left(1+q_{\mathrm{n}}^{2}\right)}{1+q_{\mathrm{N}}^{2}}\left\|\mathbf{f}_{\mathrm{N}}\right\|^{2}
$$

Ce qui établit l'inégalité (18).

La seconde inégalité (19) se démontre de façon symétrique en échangeant les rôles des lettres dans les formules ci-dessus.

Ainsi pour $\mathbf{u}^{p+1}=\mathrm{P}_{\mathrm{N}} \mathrm{Q}_{\mathrm{n}} \mathbf{u}^{p}$, on obtient :

$$
\left\|\mathbf{u}^{p+1}\right\|_{L^{2}}=\left\|\mathrm{P}_{\mathrm{N}} \mathrm{Q}_{\mathrm{n}} \mathbf{u}^{p}\right\|_{L^{2}} \leq \sqrt{\frac{1+q_{\mathrm{N}}^{2}}{1+q_{\mathrm{n}}^{2}}} q_{\mathrm{n}} \sqrt{\frac{1+q_{\mathrm{n}}^{2}}{1+q_{\mathrm{N}}^{2}}} q_{\mathrm{N}}\left\|\mathbf{u}^{p}\right\|_{L^{2}}=q_{\mathrm{n}} q_{\mathrm{N}}\left\|\mathbf{u}^{p}\right\|_{L^{2}}
$$

Ce qui démontre l'inégalité (20) et termine la démonstration.

\subsection{Vérification des hypothèses de convergence avec les ondelettes de Shannon}

Si les hypothèses (16) et (17) du théorème 3.1 sont vérifiées avec des constantes $q_{\mathrm{n}}$ et $q_{\mathrm{N}}$ telles que $q_{\mathrm{n}} q_{\mathrm{N}}<1$, alors l'algorithme converge exponentiellement comme $\left(q_{\mathrm{n}}^{p} q_{\mathrm{N}}^{p}\right)_{p \in \mathbb{N}}$. Ces deux conditions étant difficiles à vérifier 
dans le cas général, on se propose de démontrer qu'avec les ondelettes de Shannon, on peut prendre $q_{\mathrm{n}}=q_{\mathrm{N}}=\frac{3}{4}$. L'ondelette $\psi_{1}$ considérée dans cette partie aura pour transformée de Fourier ${ }^{1}$ :

$$
\widehat{\psi}_{1}(\xi)=-e^{-i \xi / 2} \chi_{[-2 \pi,-\pi] \cup[\pi, 2 \pi]}(\xi) \quad \forall \xi \in \mathbb{R}
$$

où $\chi$ désigne la fonction indicatrice. En particulier, pour tout $j, k \in \mathbb{Z}$ :

$$
\left.\mid \psi_{1} \widehat{\left(2^{j} \cdot-\right.}-k\right)(\xi) \mid=2^{-j} \chi_{\left[-2^{j+1} \pi,-2^{j} \pi\right] \cup\left[2^{j} \pi, 2^{j+1} \pi\right]}(\xi)
$$

\subsubsection{Démonstration dans le cas 2D}

Une estimation de la valeur de $q_{\mathrm{n}}$ dans la formule (16) est obtenue en comparant les normes $\left\|\mathbb{Q} \mathbf{f}_{\mathrm{n}}\right\|_{L^{2}}$ et $\left\|\mathbf{f}_{\mathrm{n}}\right\|_{L^{2}}$ pour $\mathbf{f}_{\mathrm{n}} \in \mathrm{H}_{\mathrm{n}}$.

D'après la partie $2(3)$, toute fonction $\mathbf{f}_{\mathrm{n}} \in \mathrm{H}_{\mathrm{n}}$ s'écrit :

$$
\mathbf{f}_{\mathrm{n}}=\sum_{\mathbf{j}, \mathbf{k}} d_{\mathrm{n} \mathbf{j}, \mathbf{k}} \Psi_{\mathrm{n} \mathbf{j}, \mathbf{k}} \quad \text { avec } \Psi_{\mathrm{n} \mathbf{j}, \mathbf{k}}\left(x_{1}, x_{2}\right)=\mid \begin{aligned}
& 2^{j_{1}} \psi_{1}\left(2^{j_{1}} x_{1}-k_{1}\right) \psi_{0}\left(2^{j_{2}} x_{2}-k_{2}\right) \\
& 2^{j_{2}} \psi_{0}\left(2^{j_{1}} x_{1}-k_{1}\right) \psi_{1}\left(2^{j_{2}} x_{2}-k_{2}\right)
\end{aligned}
$$

On décompose $\mathbf{f}_{\mathrm{n}}$ en utilisant (10) :

$$
\mathbf{f}_{\mathrm{n}}=\overrightarrow{\operatorname{rot}} g+\nabla p=\mathbb{P} \mathbf{f}_{\mathrm{n}}+\mathbb{Q} \mathbf{f}_{\mathrm{n}}
$$

avec $g$ et $p$ les fonctions courant et potentiel, qui sont des fonctions scalaires en dimension 2. La divergence de l'équation donne $\operatorname{div} \mathbf{f}_{\mathrm{n}}=\Delta p$, soit

$$
\Delta p\left(x_{1}, x_{2}\right)=\sum_{\mathbf{j}, \mathbf{k}}\left(2^{2 j_{1}}+2^{2 j_{2}}\right) d_{\mathrm{n} \mathbf{j}, \mathbf{k}} \psi_{0}\left(2^{j_{1}} x_{1}-k_{1}\right) \psi_{0}\left(2^{j_{2}} x_{2}-k_{2}\right)
$$

On applique la transformée de Fourier à cette équation :

$$
-|\xi|^{2} \widehat{p}\left(\xi_{1}, \xi_{2}\right)=\sum_{\mathbf{j}, \mathbf{k}}\left(2^{2 j_{1}}+2^{2 j_{2}}\right) d_{\mathbf{n} \mathbf{j}, \mathbf{k}} 2^{-j_{1}-j_{2}} \mathrm{e}^{-i 2^{-\mathbf{j}} \mathbf{k} \cdot \xi} \widehat{\psi_{0}}\left(2^{-j_{1}} \xi_{1}\right) \widehat{\psi_{0}}\left(2^{-j_{2}} \xi_{2}\right)
$$

où on a noté $2^{-\mathbf{j}} \mathbf{k}=\left(2^{-j_{1}} k_{1}, 2^{-j_{2}} k_{2}\right)$. On obtient alors :

$$
\widehat{\nabla p}\left(\xi_{1}, \xi_{2}\right)=-\frac{1}{|\xi|^{2}}\left(\sum_{\mathbf{j}, \mathbf{k}}\left(2^{j_{1}-j_{2}}+2^{j_{2}-j_{1}}\right) d_{\mathbf{n} \mathbf{j}, \mathbf{k}} \mathrm{e}^{-i 2^{-\mathbf{j}} \mathbf{k} \cdot \xi} \widehat{\psi_{0}}\left(2^{-j_{1}} \xi_{1}\right) \widehat{\psi_{0}}\left(2^{-j_{2}} \xi_{2}\right)\right)\left[\begin{array}{l}
i \xi_{1} \\
i \xi_{2}
\end{array}\right]
$$

et la norme $L^{2}$ :

$$
\|\widehat{\nabla p}\|_{L^{2}}^{2}=\iint_{\xi \in \mathbb{R}^{2}} \frac{1}{|\xi|^{2}}\left|\sum_{\mathbf{j}, \mathbf{k}}\left(2^{j_{1}-j_{2}}+2^{j_{2}-j_{1}}\right) d_{\mathrm{n} \mathbf{j}, \mathbf{k}} \mathrm{e}^{-i 2^{-\mathbf{j}} \mathbf{k} \cdot \xi} \widehat{\psi_{0}}\left(2^{-j_{1}} \xi_{1}\right) \widehat{\psi_{0}}\left(2^{-j_{2}} \xi_{2}\right)\right|^{2} d \xi
$$

Comme $\left\|\mathbb{Q} \mathbf{f}_{\mathrm{n}}\right\|_{L^{2}}^{2}=\|\nabla p\|_{L^{2}}^{2}=\frac{1}{(2 \pi)^{2}}\|\widehat{\nabla p}\|_{L^{2}}^{2}$, on cherche à minorer cette quantité à l'aide de la norme de

$$
\widehat{\mathbf{f}_{\mathrm{n}}}\left(\xi_{1}, \xi_{2}\right)=\sum_{\mathbf{j}, \mathbf{k}} d_{\mathrm{n} \mathbf{j}, \mathbf{k}} \mid \begin{aligned}
& 2^{-j_{2}} \mathrm{e}^{-i 2^{-\mathbf{j}} \mathbf{k} \cdot \xi} \widehat{\widehat{\psi_{1}}}\left(2^{-j_{1}} \xi_{1}\right) \widehat{\psi_{0}}\left(2^{-j_{2}} \xi_{2}\right) \\
& 2^{-j_{1}} \mathrm{e}^{-i 2^{-\mathbf{j}} \mathbf{k} \cdot \xi} \widehat{\psi_{0}}\left(2^{-j_{1}} \xi_{1}\right) \widehat{\psi_{1}}\left(2^{-j_{2}} \xi_{2}\right)
\end{aligned}
$$

\footnotetext{
${ }^{1}$ La transformée de Fourier d'une fonction $f \in L^{1}(\mathbb{R})$ est notée $\hat{f}(\xi)=\int_{-\infty}^{+\infty} f(x) e^{-i x \xi} d x$, et on rappelle que $f \mapsto \frac{1}{\sqrt{2 \pi}} \hat{f}$ est une isométrie de $L^{2}(\mathbb{R})$.
} 
Or, la relation de dérivation (2) entre les deux ondelettes $\psi_{1}$ et $\psi_{0}\left(\psi_{1}^{\prime}=\psi_{0}\right)$, conduit à $\widehat{\psi_{0}}(\xi)=i \xi \widehat{\psi_{1}}(\xi)(\xi \in \mathbb{R})$. On a alors la réécriture :

$$
\widehat{\mathbf{f}_{\mathrm{n}}}\left(\xi_{1}, \xi_{2}\right)=\sum_{\mathbf{j}, \mathbf{k}} d_{\mathrm{n} \mathbf{j}, \mathbf{k}} i \mathrm{e}^{-i 2^{-\mathbf{j}} \mathbf{k} \cdot \boldsymbol{\xi}} \mid \begin{array}{ll}
2^{-2 j_{2}} \xi_{2} \widehat{\psi_{1}}\left(2^{-j_{1}} \xi_{1}\right) \widehat{\psi_{1}}\left(2^{-j_{2}} \xi_{2}\right) \\
2^{-2 j_{1}} \xi_{1} \widehat{\psi_{1}}\left(2^{-j_{1}} \xi_{1}\right) \widehat{\psi_{1}}\left(2^{-j_{2}} \xi_{2}\right)
\end{array}
$$

D'où :

$$
\begin{aligned}
\left\|\widehat{\mathbf{f}_{\mathrm{n}}}\right\|_{L^{2}}^{2}= & \iint_{\xi \in \mathbb{R}^{2}} \xi_{2}^{2}\left|\sum_{\mathbf{j}, \mathbf{k}} 2^{-2 j_{2}} d_{\mathrm{n} \mathbf{j}, \mathbf{k}} \mathrm{e}^{-i 2^{-\mathbf{j}} \mathbf{k} \cdot \xi} \widehat{\psi_{1}}\left(2^{-j_{1}} \xi_{1}\right) \widehat{\psi_{1}}\left(2^{-j_{2}} \xi_{2}\right)\right|^{2} d \xi \\
& +\iint_{\xi \in \mathbb{R}^{2}} \xi_{1}^{2}\left|\sum_{\mathbf{j}, \mathbf{k}} 2^{-2 j_{1}} d_{\mathbf{n} \mathbf{j}, \mathbf{k}} \mathrm{e}^{-i 2^{-\mathbf{j}} \mathbf{k} \cdot \xi} \widehat{\psi_{1}}\left(2^{-j_{1}} \xi_{1}\right) \widehat{\psi_{1}}\left(2^{-j_{2}} \xi_{2}\right)\right|^{2} d \xi
\end{aligned}
$$

On se place dans le quart de plan $\mathbb{R}^{+} \times \mathbb{R}^{+}$(sachant que les trois autres quarts du plan $\mathbb{R}^{2}$ vérifieront les mêmes encadrements) et on considère le rectangle $R_{\ell}=\left[2^{\ell_{1}} \pi, 2^{\ell_{1}+1} \pi\right] \times\left[2^{\ell_{2}} \pi, 2^{\ell_{2}+1} \pi\right]$ pour $\ell=\left(\ell_{1}, \ell_{2}\right) \in \mathbb{Z}^{2}$. En utilisant (21), on obtient :

$$
\left\|\widehat{\mathbf{f}}_{\mathrm{n}}\right\|_{L^{2}\left(\mathrm{R}_{\ell}\right)}^{2}=\iint_{\xi \in \mathrm{R}_{\ell}}\left(2^{-4 \ell_{1}} \xi_{1}^{2}+2^{-4 \ell_{2}} \xi_{2}^{2}\right)\left|\sum_{\mathbf{k}} d_{\mathrm{n} \ell, \mathbf{k}} \mathrm{e}^{-i 2^{-\ell} \mathbf{k} \cdot \xi} \widehat{\psi_{1}}\left(2^{-\ell_{1}} \xi_{1}\right) \widehat{\psi_{1}}\left(2^{-\ell_{2}} \xi_{2}\right)\right|^{2} d \xi
$$

De la même manière,

$$
\|\widehat{\nabla p}\|_{L^{2}\left(\mathrm{R}_{\ell}\right)}^{2}=\iint_{\xi \in \mathrm{R}_{\ell}}\left(2^{-2 \ell_{1}}+2^{-2 \ell_{2}}\right)^{2} \frac{\xi_{1}^{2} \xi_{2}^{2}}{|\xi|^{2}}\left|\sum_{\mathbf{k}} d_{\mathrm{n} \ell, \mathbf{k}} \mathrm{e}^{-i 2^{-\ell} \mathbf{k} \cdot \xi} \widehat{\psi_{1}}\left(2^{-\ell_{1}} \xi_{1}\right) \widehat{\psi_{1}}\left(2^{-\ell_{2}} \xi_{2}\right)\right|^{2} d \xi
$$

Il faut donc comparer

$$
q_{1}(\xi)=\left(2^{-2 \ell_{1}}+2^{-2 \ell_{2}}\right)^{2} \frac{\xi_{1}^{2} \xi_{2}^{2}}{|\xi|^{2}} \text { avec } q_{2}(\xi)=\left(2^{-4 \ell_{1}} \xi_{1}^{2}+2^{-4 \ell_{2}} \xi_{2}^{2}\right)
$$

pour $\xi=\left(\xi_{1}, \xi_{2}\right) \in\left[2^{\ell_{1}} \pi, 2^{\ell_{1}+1} \pi\right] \times\left[2^{\ell_{2}} \pi, 2^{\ell_{2}+1} \pi\right]$.

Si on pose

$$
y=\left(\frac{2^{-\ell_{1}} \xi_{1}}{2^{-\ell_{2}} \xi_{2}}\right)^{2} \in\left[\frac{1}{4}, 4\right] \text { pour } \xi \in \mathrm{R}_{\ell}
$$

on obtient

$$
\frac{q_{2}}{q_{1}}(\xi)=\frac{2^{2 \ell_{1}+2 \ell_{2}}}{\left(2^{2 \ell_{1}}+2^{2 \ell_{2}}\right)^{2}}\left(y+\frac{1}{y}+2^{2 \ell_{1}-2 \ell_{2}}+2^{2 \ell_{2}-2 \ell_{1}}\right)
$$

Ainsi, le rapport $q_{2}(\xi) / q_{1}(\xi)$ dont on cherche un majorant sur le rectangle $\mathrm{R}_{\ell}$ est minimum en $y=1$, donc sur la droite $\left\{2^{-\ell_{1}} \xi_{1}=2^{-\ell_{2}} \xi_{2}\right\}$, et atteint son maximum sur le rectangle $\mathrm{R}_{\ell}$ aux deux coins $\left(\xi_{1}, \xi_{2}\right)=\left(2^{\ell_{1}} \pi, 2^{\ell_{2}+1} \pi\right)$ et $\left(\xi_{1}, \xi_{2}\right)=\left(2^{\ell_{1}+1} \pi, 2^{\ell_{2}} \pi\right)$ du rectangle $\mathrm{R}_{\ell}$ où $y$ vaut respectivement $\frac{1}{4}$ et 4 . Par symétrie sur les variables $\ell_{1}$ et $\ell_{2}$, on n'en considère qu'un, $\left(2^{\ell_{1}+1} \pi, 2^{\ell_{2}} \pi\right)$. Le rapport $q_{2}(\xi) / q_{1}(\xi)$ vaut alors :

$$
\frac{q_{2}}{q_{1}}\left(2^{\ell_{1}+1} \pi, 2^{\ell_{2}} \pi\right)=\frac{2^{2 \ell_{1}+2 \ell_{2}+2}+2^{4 \ell_{1}}+2^{4 \ell_{2}}+2^{2 \ell_{1}+2 \ell_{2}-2}}{\left(2^{2 \ell_{1}}+2^{2 \ell_{2}}\right)^{2}}
$$

qui donne en posant $2^{\ell_{1}}=\rho \cos \theta$ et $2^{\ell_{2}}=\rho \sin \theta$,

$$
\frac{q_{2}}{q_{1}}\left(2^{\ell_{1}+1} \pi, 2^{\ell_{2}} \pi\right)=1+\frac{9}{16} \sin ^{2}(2 \theta)
$$


qui est maximum pour $\theta=\pi / 4$ c'est-à-dire $\ell_{1}=\ell_{2}$.

On a donc $\forall \ell \in \mathbb{Z}^{2}, \forall\left(\xi_{1}, \xi_{2}\right) \in \mathrm{R}_{\ell}, q_{1}(\xi) \geq \frac{16}{25} q_{2}(\xi)$.

En réintroduisant cette majoration dans les intégrales,

$$
\forall \ell \in \mathbb{Z}^{2},\|\widehat{\nabla p}\|_{L^{2}\left(\mathrm{R}_{\ell}\right)}^{2} \geq \frac{16}{25}\left\|\widehat{\mathbf{f}}_{\mathrm{n}}\right\|_{L^{2}\left(\mathrm{R}_{\ell}\right)}^{2}
$$

et en sommant sur tous les $\ell \in \mathbb{Z}^{2}$ et sur les quatre quarts du plan, on obtient :

$$
\|\widehat{\nabla p}\|_{L^{2}\left(\mathbb{R}^{2}\right)}^{2} \geq \frac{16}{25}\left\|\widehat{\mathbf{f}_{\mathrm{n}}}\right\|_{L^{2}\left(\mathbb{R}^{2}\right)}^{2} \quad \text { ou encore } \quad\|\nabla p\|_{L^{2}\left(\mathbb{R}^{2}\right)}^{2} \geq \frac{16}{25}\left\|\mathbf{f}_{\mathrm{n}}\right\|_{L^{2}\left(\mathbb{R}^{2}\right)}^{2}
$$

Ainsi, $\left\|\mathbf{f}_{\mathrm{n}}\right\|_{L^{2}}^{2}=\left\|\mathbb{P} \mathbf{f}_{\mathrm{n}}\right\|_{L^{2}}^{2}+\left\|\mathbb{Q} \mathbf{f}_{\mathrm{n}}\right\|_{L^{2}}^{2} \leq \frac{25}{16}\left\|\mathbb{Q} \mathbf{f}_{\mathrm{n}}\right\|_{L^{2}}^{2}$.

Finalement $\left\|\mathbb{P} \mathbf{f}_{\mathrm{n}}\right\|_{L^{2}}^{2} \leq \frac{9}{16}\left\|\mathbb{Q} \mathbf{f}_{\mathrm{n}}\right\|_{L^{2}}^{2}$, et la constante $q_{\mathrm{n}}=3 / 4$ convient.

De manière analogue, pour $\mathbf{f}_{\mathrm{N}} \in \mathrm{H}_{\mathrm{N}}$, on démontre que

$$
\left\|\mathbb{Q} \mathbf{f}_{\mathrm{N}}\right\|_{L^{2}}^{2} \leq \frac{9}{16}\left\|\mathbb{P} \mathbf{f}_{\mathrm{N}}\right\|_{L^{2}}^{2}
$$

et on peut prendre $q_{\mathrm{N}}=3 / 4$. Ainsi le produit $q_{\mathrm{n}} q_{\mathrm{N}}=9 / 16$ est strictement inférieur à 1 et l'algorithme converge. Il est remarquable que ce taux corresponde assez bien à celui qui est obtenu expérimentalement avec des ondelettes splines à faible nombre de moments nuls, qui est d'environ 0.5 [4].

\subsubsection{Démonstration dans le cas $3 D$}

Le cas de la convergence 3D est abordé de façon similaire, mais ici il faut distinguer les cas des espaces $\mathrm{H}_{\mathrm{n}}$ et $\mathrm{H}_{\mathrm{N}}$.

Soit $\mathbf{f}_{\mathrm{n}} \in \mathrm{H}_{\mathrm{n}}$, on a :

$$
\mathbf{f}_{\mathrm{n}}=\sum_{\mathbf{j}, \mathbf{k}} d_{\mathrm{n} \mathbf{j}, \mathbf{k}} \Psi_{\mathrm{n} \mathbf{j}, \mathbf{k}}
$$

les $\Psi_{\mathrm{n} \mathbf{j}, \mathbf{k}}$ étant définies à la formule (5). Comme en $2 \mathrm{D}$, on décompose $\mathbf{f}_{\mathrm{n}}$ suivant (10) :

$$
\mathbf{f}_{\mathrm{n}}=\overrightarrow{\operatorname{rot}} g+\nabla p=\mathbb{P} \mathbf{f}_{\mathrm{n}}+\mathbb{Q} \mathbf{f}_{\mathrm{n}}
$$

et par application de la transformée de Fourier :

$$
\begin{aligned}
\widehat{\mathbb{Q} \mathbf{f}_{\mathrm{n}}}\left(\xi_{1}, \xi_{2}, \xi_{3}\right) & =\widehat{\nabla p}\left(\xi_{1}, \xi_{2}, \xi_{3}\right) \\
& =-\frac{1}{|\xi|^{2}}\left(\sum_{\mathbf{j}, \mathbf{k}}\left(\frac{2^{2 j_{1}}+2^{2 j_{2}}+2^{2 j_{3}}}{2^{j_{1}+j_{2}+j_{3}}}\right) d_{\mathrm{n} \mathbf{j}, \mathbf{k}} \mathrm{e}^{-i 2^{-\mathbf{j}} \mathbf{k} \cdot \xi} \widehat{\psi_{0}}\left(2^{-j_{1}} \xi_{1}\right) \widehat{\psi_{0}}\left(2^{-j_{2}} \xi_{2}\right) \widehat{\psi_{0}}\left(2^{-j_{3}} \xi_{3}\right)\right)\left[\begin{array}{c}
i \xi_{1} \\
i \xi_{2} \\
i \xi_{3}
\end{array}\right]
\end{aligned}
$$

dont il faut comparer la norme $L^{2}$ avec celle de

$$
\widehat{\mathbf{f}_{\mathrm{n}}}\left(\xi_{1}, \xi_{2}, \xi_{3}\right)=i \sum_{\mathbf{j}, \mathbf{k}} d_{\mathrm{n} \mathbf{j}, \mathbf{k}} 2^{-2 j_{1}-2 j_{2}-2 j_{3}} \mathrm{e}^{-i 2^{-\mathbf{j}} \mathbf{k} \cdot \xi} \mid \begin{aligned}
& 2^{2 j_{1}} \xi_{2} \xi_{3} \widehat{\psi_{1}}\left(2^{-j_{1}} \xi_{1}\right) \widehat{\psi_{1}}\left(2^{-j_{2}} \xi_{2}\right) \widehat{\psi_{1}}\left(2^{-j_{3}} \xi_{3}\right) \\
& 2^{2 j_{2}} \xi_{1} \xi_{3} \widehat{\psi_{1}}\left(2^{-j_{1}} \xi_{1}\right) \widehat{\psi_{1}}\left(2^{-j_{2}} \xi_{2}\right) \widehat{\widehat{\psi_{1}}}\left(2^{-j_{3}} \xi_{3}\right) \\
& 2^{2 j_{3}} \xi_{1} \xi_{2} \widehat{\psi_{1}}\left(2^{-j_{1}} \xi_{1}\right) \widehat{\psi_{1}}\left(2^{-j_{2}} \xi_{2}\right) \widehat{\psi_{1}}\left(2^{-j_{3}} \xi_{3}\right)
\end{aligned}
$$


Pour effectuer cette comparaison dans le cas des ondelettes de Shannon, on découpe le domaine d'intégration $\mathbb{R}^{3}$ en pavés $\mathrm{R}_{\ell, \varepsilon}=\left[2^{\ell_{1}} \pi \varepsilon_{1}, 2^{\ell_{1}+1} \pi \varepsilon_{1}\right] \times\left[2^{\ell_{2}} \pi \varepsilon_{2}, 2^{\ell_{2}+1} \pi \varepsilon_{2}\right] \times\left[2^{\ell_{3}} \pi \varepsilon_{3}, 2^{\ell_{3}+1} \pi \varepsilon_{3}\right]$ pour $\ell=\left(\ell_{1}, \ell_{2}, \ell_{3}\right) \in \mathbb{Z}^{3}$ et $\varepsilon \in\{-1,1\}^{3}$. Par symétrie, on se restreint au cas $\varepsilon=\{1,1,1\}$. Sur ces pavés, on trouve comme normes :

$$
\begin{array}{r}
\left\|\widehat{\mathbf{f}_{\mathrm{n}}}\right\|_{L^{2}\left(\mathrm{R}_{\ell}\right)}^{2}=\iiint_{\xi \in \mathrm{R}_{\ell}}\left(2^{-4 \ell_{2}} \xi_{2}^{2} 2^{-4 \ell_{3}} \xi_{3}^{2}+2^{-4 \ell_{1}} \xi_{1}^{2} 2^{-4 \ell_{3}} \xi_{3}^{2}+2^{-4 \ell_{1}} \xi_{1}^{2} 2^{-4 \ell_{2}} \xi_{2}^{2}\right) \\
\left|\sum_{\mathbf{k}} d_{\mathrm{n} \ell, \mathbf{k}} \mathrm{e}^{-i 2^{-\ell} \mathbf{k} \cdot \xi} \widehat{\psi_{1}}\left(2^{-\ell_{1}} \xi_{1}\right) \widehat{\psi_{1}}\left(2^{-\ell_{2}} \xi_{2}\right) \widehat{\psi}_{1}\left(2^{-\ell_{3}} \xi_{3}\right)\right|^{2} d \xi
\end{array}
$$

et

$$
\begin{gathered}
\left\|\widehat{\mathbb{Q} \mathbf{f}_{\mathrm{n}}}\right\|_{L^{2}\left(\mathrm{R}_{\ell}\right)}^{2}=\iiint_{\xi \in \mathrm{R}_{\ell}}\left(\frac{2^{2 \ell_{1}}+2^{2 \ell_{2}}+2^{2 \ell_{3}}}{2^{2 \ell_{1}+2 \ell_{2}+2 \ell_{3}}}\right)^{2} \frac{\xi_{1}^{2} \xi_{2}^{2} \xi_{3}^{2}}{|\xi|^{2}} \\
\left|\sum_{\mathbf{k}} d_{\mathrm{n} \ell, \mathbf{k}} \mathrm{e}^{-i 2^{-\ell} \mathbf{k} \cdot \xi} \widehat{\psi_{1}}\left(2^{-\ell_{1}} \xi_{1}\right) \widehat{\psi_{1}}\left(2^{-\ell_{2}} \xi_{2}\right) \widehat{\psi_{1}}\left(2^{-\ell_{3}} \xi_{3}\right)\right|^{2} d \xi
\end{gathered}
$$

On pose

et

$$
q_{1}(\xi, \ell)=\left(\frac{2^{2 \ell_{1}}+2^{2 \ell_{2}}+2^{2 \ell_{3}}}{2^{2 \ell_{1}+2 \ell_{2}+2 \ell_{3}}}\right)^{2} \frac{\xi_{1}^{2} \xi_{2}^{2} \xi_{3}^{2}}{|\xi|^{2}}
$$

$$
q_{2}(\xi, \ell)=\left(2^{-4 \ell_{2}} \xi_{2}^{2} 2^{-4 \ell_{3}} \xi_{3}^{2}+2^{-4 \ell_{1}} \xi_{1}^{2} 2^{-4 \ell_{3}} \xi_{3}^{2}+2^{-4 \ell_{1}} \xi_{1}^{2} 2^{-4 \ell_{2}} \xi_{2}^{2}\right)
$$

On introduit $x_{i}=2^{-\ell_{i}} \xi_{i} / \pi$, pour $i=1,2,3$, qui vérifie $x_{i} \in[1,2]$.

On cherche alors un majorant de $\frac{\left\|\widehat{\mathrm{f}}_{\mathrm{n}}\right\|_{L^{2}\left(\mathrm{R}_{\ell}\right)}^{2}}{\| \widehat{\mathbb{Q} \mathbf{f}_{\mathrm{n}} \|_{L^{2}\left(\mathrm{R}_{\ell}\right)}^{2}}}$ grâce à :

$$
\begin{aligned}
E(\mathbf{x}, \ell)=\frac{q_{2}(\xi, \ell)}{q_{1}(\xi, \ell)} & =\frac{\left(2^{2 \ell_{1}} x_{1}^{2}+2^{2 \ell_{2}} x_{2}^{2}+2^{2 \ell_{3}} x_{3}^{2}\right)\left(2^{2 \ell_{1}} x_{2}^{2} x_{3}^{2}+2^{2 \ell_{2}} x_{1}^{2} x_{3}^{2}+2^{2 \ell_{3}} x_{1}^{2} x_{2}^{2}\right)}{\left(2^{2 \ell_{1}}+2^{2 \ell_{2}}+2^{2 \ell_{3}}\right)^{2} x_{1}^{2} x_{2}^{2} x_{3}^{2}} \\
& =\frac{\left(2^{2 \ell_{1}} x_{1}^{2}+2^{2 \ell_{2}} x_{2}^{2}+2^{2 \ell_{3}} x_{3}^{2}\right)\left(2^{2 \ell_{1}} \frac{1}{x_{1}^{2}}+2^{2 \ell_{2}} \frac{1}{x_{2}^{2}}+2^{2 \ell_{3}} \frac{1}{x_{3}^{2}}\right)}{\left(2^{2 \ell_{1}}+2^{2 \ell_{2}}+2^{2 \ell_{3}}\right)^{2}}
\end{aligned}
$$

On pose alors, pour $i=1,2,3: \alpha_{i}=\frac{2^{\ell_{i}}}{\left(2^{2 \ell_{1}}+2^{2 \ell_{2}}+2^{2 \ell_{3}}\right)^{1 / 2}}$ et $z_{i}=x_{i}^{2}$. On doit alors maximiser :

$$
E(\mathbf{x}, \ell)=F\left(\mathbf{z}, \alpha_{i}\right)=\left(\sum_{i=1}^{3} \alpha_{i}^{2} z_{i}\right)\left(\sum_{i=1}^{3} \frac{\alpha_{i}^{2}}{z_{i}}\right)
$$

pour $\mathbf{z}=\left(z_{1}, z_{2}, z_{3}\right) \in[1,4]^{3}$, sous la contrainte $\sum_{i=1}^{3} \alpha_{i}^{2}=1$. On reconnaît une inégalité de Kantorovitch qui conduit, à $\mathbf{z}$ fixé, à :

$$
\max _{\alpha_{i}, \sum \alpha_{i}^{2}=1} F\left(\mathbf{z}, \alpha_{i}\right)=\frac{1}{4}\left(\sqrt{\frac{\min z_{i}}{\max z_{i}}}+\sqrt{\frac{\max z_{i}}{\min z_{i}}}\right)^{2}
$$

Donc pour $\mathbf{z} \in[1,4]^{3}$, on a :

$$
\max _{\mathbf{z}} \max _{\alpha_{i}, \sum \alpha_{i}^{2}=1} F\left(\mathbf{z}, \alpha_{i}\right)=\frac{1}{4}\left(\sqrt{\frac{1}{4}}+\sqrt{\frac{4}{1}}\right)^{2}=\frac{25}{16}
$$


Finalement $E(\mathbf{x}, \ell) \leq \frac{25}{16}$ et $\left\|\mathbb{P} \mathbf{f}_{\mathrm{n}}\right\|_{L^{2}}^{2} \leq \frac{9}{16}\left\|\mathbb{Q} \mathbf{f}_{\mathrm{n}}\right\|_{L^{2}}^{2}$.

Ainsi on peut prendre $q_{n}=\frac{3}{4}$.

Soit maintenant $\mathbf{f}_{\mathrm{N}} \in \mathrm{H}_{\mathrm{N}}$, on a :

$$
\mathbf{f}_{\mathrm{N}}=\sum_{\mathbf{j}, \mathbf{k}} d_{\mathrm{N} 1, \mathbf{j}, \mathbf{k}} \Psi_{\mathrm{N} 1, \mathbf{j}, \mathbf{k}}+d_{\mathrm{N} 2, \mathbf{j}, \mathbf{k}} \Psi_{\mathrm{N} 2, \mathbf{j}, \mathbf{k}}+d_{\mathrm{N} 3, \mathbf{j}, \mathbf{k}} \Psi_{\mathrm{N} 3, \mathbf{j}, \mathbf{k}}
$$

les fonctions $\Psi_{\mathrm{N} 1, \mathbf{j}, \mathbf{k}}$ étant définies à la formule (9). Pour calculer le projeté $\mathbb{Q} \mathbf{f}_{\mathrm{N}}$ de $\mathbf{f}_{\mathrm{N}}$ on utilise la transformée de Fourier :

$$
\widehat{\mathbf{f}_{\mathrm{N}}}(\xi)=i \sum_{\mathbf{j}, \mathbf{k}} \mathrm{e}^{-i 2^{-\mathbf{j}} \mathbf{k} \cdot \xi} \mid \begin{aligned}
& 2^{-2 j_{1}} \xi_{1}\left(2^{-j_{2}} d_{\mathrm{N} 2, \mathbf{j}, \mathbf{k}}-2^{-j_{3}} d_{\mathrm{N} 3, \mathbf{j}, \mathbf{k}}\right) \widehat{\psi_{1}}\left(2^{-j_{1}} \xi_{1}\right) \widehat{\psi_{1}}\left(2^{-j_{2}} \xi_{2}\right) \widehat{\psi_{1}}\left(2^{-j_{3}} \xi_{3}\right) \\
& 2^{-2 j_{2}} \xi_{2}\left(2^{-j_{3}} d_{\mathrm{N} 3, \mathbf{j}, \mathbf{k}}-2^{-j_{1}} d_{\mathrm{N} 1, \mathbf{j}, \mathbf{k}}\right) \widehat{\psi_{1}}\left(2^{-j_{1}} \xi_{1}\right) \widehat{\psi_{1}}\left(2^{-j_{2}} \xi_{2}\right) \widehat{\psi_{1}}\left(2^{-j_{3}} \xi_{3}\right) \\
& 2^{-2 j_{3}} \xi_{3}\left(2^{-j_{1}} d_{\mathrm{N} 1, \mathbf{j}, \mathbf{k}}-2^{-j_{2}} d_{\mathrm{N} 2, \mathbf{j}, \mathbf{k}}\right) \widehat{\psi_{1}}\left(2^{-j_{1}} \xi_{1}\right) \widehat{\psi_{1}}\left(2^{-j_{2}} \xi_{2}\right) \widehat{\psi_{1}}\left(2^{-j_{3}} \xi_{3}\right)
\end{aligned}
$$

et comme $-|\xi|^{2} \widehat{p}=\sum_{m=1}^{3} i \xi_{m}\left(\widehat{\mathbf{f}_{\mathrm{N}}}\right)_{m}$ (on utilise de nouveau (10) pour $\mathbf{f}_{\mathrm{N}}$ ), on trouve :

$$
\begin{array}{r}
\widehat{\mathbb{Q} \mathbf{f}_{\mathrm{N}}}(\xi)=-\frac{1}{|\xi|^{2}}\left(\sum _ { \mathbf { j } , \mathbf { k } } \left[2^{-2 j_{1}} \xi_{1}^{2}\left(2^{-j_{2}} d_{\mathrm{N} 2, \mathbf{j}, \mathbf{k}}-2^{-j_{3}} d_{\mathrm{N} 3, \mathbf{j}, \mathbf{k}}\right)+2^{-2 j_{2}} \xi_{2}^{2}\left(2^{-j_{3}} d_{\mathrm{N} 3, \mathbf{j}, \mathbf{k}}-2^{-j_{1}} d_{\mathrm{N} 1, \mathbf{j}, \mathbf{k}}\right)\right.\right. \\
\left.\left.\quad+2^{-2 j_{3}} \xi_{3}^{2}\left(2^{-j_{1}} d_{\mathrm{N} 1, \mathbf{j}, \mathbf{k}}-2^{-j_{2}} d_{\mathrm{N} 2, \mathbf{j}, \mathbf{k}}\right)\right] i \mathrm{e}^{-i 2^{-\mathbf{j}} \mathbf{k} \cdot \xi} \widehat{\psi_{1}}\left(2^{-j_{1}} \xi_{1}\right) \widehat{\psi_{1}}\left(2^{-j_{2}} \xi_{2}\right) \widehat{\psi_{1}}\left(2^{-j_{3}} \xi_{3}\right)\right)\left[\begin{array}{c}
i \xi_{1} \\
i \xi_{2} \\
i \xi_{3}
\end{array}\right]
\end{array}
$$

Afin de calculer les normes $L^{2}$ sur le pavé $\mathrm{R}_{\ell}$, on pose, pour $m=1,2,3$ :

$$
X_{m}(\xi)=\sum_{\mathbf{k}}\left(2^{-\ell_{m+1}[3]} d_{\mathrm{N} m+1[3], \ell, \mathbf{k}}-2^{-\ell_{m+2[3]}} d_{\mathrm{N} m+2[3], \ell, \mathbf{k}}\right) \mathrm{e}^{-i 2^{-\ell} \mathbf{k} \cdot \xi} \widehat{\psi_{1}}\left(2^{-\ell_{1}} \xi_{1}\right) \widehat{\psi_{1}}\left(2^{-\ell_{2}} \xi_{2}\right) \widehat{\psi_{1}}\left(2^{-\ell_{3}} \xi_{3}\right)
$$

On remarque tout d'abord que $X_{1}(\xi)+X_{2}(\xi)+X_{3}(\xi)=0$. Et on obtient les normes :

$$
\left\|\widehat{\mathbf{f}_{\mathrm{N}}}\right\|_{L^{2}\left(\mathrm{R}_{\ell}\right)}^{2}=\iiint_{\xi \in \mathrm{R}_{\ell}}\left(2^{-4 \ell_{1}} \xi_{1}^{2}\left|X_{1}(\xi)\right|^{2}+2^{-4 \ell_{2}} \xi_{2}^{2}\left|X_{2}(\xi)\right|^{2}+2^{-4 \ell_{3}} \xi_{3}^{2}\left|X_{3}(\xi)\right|^{2}\right) d \xi
$$

et

$$
\left\|\widehat{\mathbb{Q} \mathbf{f}_{\mathrm{N}}}\right\|_{L^{2}\left(\mathrm{R}_{\ell}\right)}^{2}=\iiint_{\xi \in \mathrm{R}_{\ell}} \frac{1}{|\xi|^{2}}\left|2^{-2 \ell_{1}} \xi_{1}^{2} X_{1}(\xi)+2^{-2 \ell_{2}} \xi_{2}^{2} X_{2}(\xi)+2^{-2 \ell_{3}} \xi_{3}^{2} X_{3}(\xi)\right|^{2} d \xi
$$

On compare les deux quantités sous le signe intégrale. On pose $X_{m}(\xi)=a_{m}(\xi)+i b_{m}(\xi)$. Cela permet de décorréler partie réelle et partie imaginaire, qui de fait, vont vérifier la même inégalité. On va comparer :

$$
q_{1}(\mathbf{a})=\frac{1}{|\xi|^{2}}\left(2^{-2 \ell_{1}} \xi_{1}^{2} a_{1}+2^{-2 \ell_{2}} \xi_{2}^{2} a_{2}+2^{-2 \ell_{3}} \xi_{3}^{2} a_{3}\right)^{2}
$$

et

$$
q_{2}(\mathbf{a})=2^{-4 \ell_{1}} \xi_{1}^{2} a_{1}^{2}+2^{-4 \ell_{2}} \xi_{2}^{2} a_{2}^{2}+2^{-4 \ell_{3}} \xi_{3}^{2} a_{3}^{2}
$$

avec $a_{1}+a_{2}+a_{3}=0$, à $\ell$ et $\xi$ fixés.

Afin de majorer le quotient $q_{1}(\mathbf{a}) / q_{2}(\mathbf{a})$, on introduit un problème de maximisation sous contraintes. On pose, pour $i=1,2,3, \alpha_{i}=2^{-2 \ell_{i}} \xi_{i} a_{i}$. On travaille sur la sphère unité en posant $q_{2}(\mathbf{a})=\sum \alpha_{i}^{2}=1$. Alors,

$$
q_{1}(\mathbf{a})=\frac{1}{|\xi|^{2}}(J(\alpha))^{2}
$$


avec $J(\alpha)=\sum_{i=1}^{3} \xi_{i} \alpha_{i}$ la fonctionnelle dont on cherche les points d'extrêma $\alpha_{\mathrm{m}}$, et dont le gradient vaut $\nabla J(\alpha)=\xi$. Les $\alpha_{i}$ vérifient une deuxième contrainte donnée par $\sum_{i=1}^{3} a_{i}=\sum_{i=1}^{3} 2^{2 \ell_{i}} \xi_{i}^{-1} \alpha_{i}=0$, c'est-à-dire que $\alpha_{\mathrm{m}}$ appartient au plan $\mathcal{P}$ passant par 0 et de vecteur normal $\mathbf{n}=\left(2^{2 \ell_{i}} \xi_{i}^{-1}\right)_{i=1,2,3}$.

Cette maximisation de $J$ sous contraintes implique l'existence de deux multiplicateurs de Lagrange $\tau$ et $\sigma$ tels que :

$$
\nabla J\left(\alpha_{m}\right)+\tau \mathbf{n}+2 \sigma \alpha_{m}=0
$$

En utilisant les relations $\langle\alpha, \mathbf{n}\rangle=0$ et $\|\alpha\|^{2}=1$, on obtient :

$$
\tau=-\frac{<\mathbf{n}, \xi>}{\|\mathbf{n}\|^{2}} \text { et } \quad 2 \sigma=-<\alpha_{m}, \xi>
$$

D'où :

$$
\left(J\left(\alpha_{m}\right)\right)^{2}=\left(<\alpha_{m}, \xi>\right)^{2}=\|\xi\|^{2}-\frac{<\mathbf{n}, \xi>^{2}}{\|\mathbf{n}\|^{2}}
$$

et on trouve la majoration :

$$
q_{1}(\mathbf{a}) \leq \frac{\|\mathbf{n}\|^{2}\|\xi\|^{2}-<\mathbf{n}, \xi>^{2}}{\|\mathbf{n}\|^{2}\|\xi\|^{2}}=1-\frac{\left(\sum_{i=1}^{3} 2^{2 \ell_{i}}\right)^{2}}{\left(\sum_{i=1}^{3} 2^{2 \ell_{i}} x_{i}^{-2}\right)\left(\sum_{i=1}^{3} 2^{2 \ell_{i}} x_{i}^{2}\right)}
$$

où on a de nouveau posé $x_{i}=2^{-\ell_{i}} \xi_{i} / \pi \in[1,2]$.

On utilise alors l'inégalité de Kantorovitch sur les $2^{2 \ell_{i}}$ qui conduit à

$$
q_{1}(\mathbf{a}) \leq 1-4\left(\frac{\min x_{i}}{\max x_{i}}+\frac{\max x_{i}}{\min x_{i}}\right)^{-2} \leq \frac{9}{25}
$$

Ce qui conduit à $\left\|\mathbb{Q} \mathbf{f}_{\mathrm{N}}\right\|^{2} \leq \frac{9}{25}\left\|\mathbf{f}_{\mathrm{N}}\right\|^{2}$.

D'où $\left\|\mathbb{Q} \mathbf{f}_{N}\right\|^{2} \leq \frac{9}{16}\left\|\mathbb{P} \mathbf{f}_{N}\right\|^{2}$ et on peut prendre $q_{N}=\frac{3}{4}$.

La démonstration dans le cas $n$-dimensionnel et avec les ondelettes à divergence nulle présentées dans [4], se démontre de façon analogue au cas 3-dimensionnel.

\section{Conclusion}

Dans cet article nous avons étudié la convergence d'un algorithme par ondelettes, pour la décomposition de Helmholtz de fonctions vectorielles sur $\mathbb{R}^{n}$. La décomposition repose sur des bases stables, localisées en espace : contrairement aux méthodes basées sur la transformée de Fourier, notre décomposition est extensible à des domaines $\Omega$ plus généraux. Cette méthode ouvre ainsi des perspectives très intéressantes en vue de la résolution directe des équations de Navier-Stokes incompressibles sur bases d'ondelettes $[4,5]$.

Bien sûr, l'algorithme proposé pose encore plusieurs questions : sur le plan théorique tout d'abord, pour démontrer la convergence avec des ondelettes plus générales que les ondelettes de Shannon (convergence vérifiée expérimentalement [4]); pour optimiser la vitesse de convergence ensuite, où se pose le problème du choix des ondelettes et des espaces complémentaires. Sur ce point, un travail en cours s'appuie sur le principe des paquets d'ondelettes pour affiner la localisation en fréquence des ondelettes utilisées [4]. L'utilisation de cette méthode dans un cadre adaptatif va aussi demander de légères modifications de l'algorithme, et une implémentation adaptée. 


\section{RÉFÉRENCES}

[1] K. Bittner et K. Urban, On interpolatory divergence-free wavelets, à paraître, Mathematics of Computation, 2006.

[2] A.J. Chorin, et J.E. Marsden, A Mathematical Introduction to Fluid Mechanics, 3rd ed., Springer, 1993.

[3] A. Cohen, I. Daubechies et J.C. Feauveau, Biorthogonal bases of compactly supported wavelets, Comm. Pure Appl. Math., 45, 485-560, 1992.

[4] E. Deriaz, Ondelettes pour la Simulation des Écoulements Fluides Incompressibles en Turbulence, Thèse de doctorat en mathématiques appliquées de l'INP Grenoble, Mars 2006.

[5] E. Deriaz et V. Perrier, Divergence-free and curl-free wavelets in $2 D$ and $3 D$, application to turbulent flows, J. of Turbulence, Volume 7, Number 3, pages 1-37, 2006.

[6] V. Girault, P.A. Raviart, Finite element methods for Navier-Stokes equations, Springer-Verlag Berlin, 1986.

[7] J.P. Kahane, P.G. Lemarié, Séries de Fourier et Ondelettes, Cassini, Nouvelle Bibliothèque Mathématique, 1998.

[8] P.G. Lemarié-Rieusset, Analyses multi-résolutions non orthogonales, commutation entre projecteurs et dérivation et ondelettes vecteurs à divergence nulle, Revista Matemática Iberoamericana, 8(2) : 221-236, 1992.

[9] S. Mallat, Une exploration des signaux en ondelettes, Éditions de l'École Polytechique, Novembre 2000.

[10] J.C. Nédélec, Acoustic and Electromagnic Equations Integral Representation for Harmonic Problems, Springer, New-York, 2001.

[11] O. Pironneau, Méthodes d'éléments finis pour les fluides, Masson, 1988.

[12] K. Urban, Using divergence-free wavelets for the numerical solution of the Stokes problem, AMLI'96 : Proceedings of the Conference on Algebraic Multilevel Iteration Methods with Applications, 2 : 261-277, University of Nijmegen, The Netherlands, 1996.

[13] K. Urban, Wavelet Bases in H(div) and H(curl), Mathematics of Computation 70(234) : 739-766, 2000.

[14] K. Urban, Wavelets in Numerical Simulation, Springer, 2002. 